Document downloaded from:

http://hdl.handle.net/10251/150645

This paper must be cited as:

Rodriguez, I.; Lequerica, JL.; Berjano, E.; Herrero, M.; Hornero, F. (2007). Esophageal temperature monitoring during radiofrequency catheter ablation: experimental study based on an agar phantom model. Physiological Measurement. 28(5):453-463.

https://doi.org/10.1088/0967-3334/28/5/001

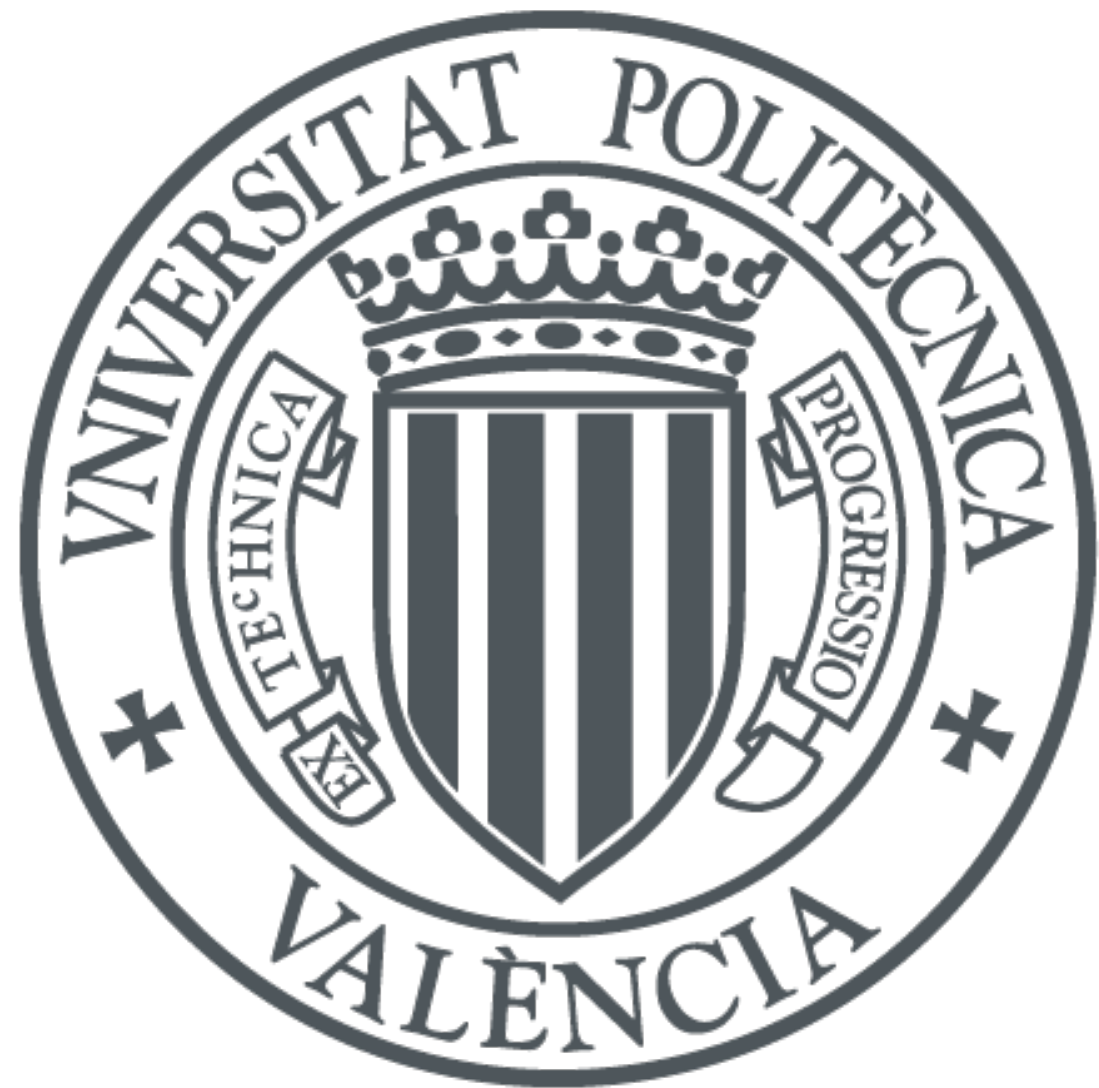

The final publication is available at

https://doi.org/10.1088/0967-3334/28/5/001

Copyright IOP Publishing

Additional Information 


\section{Esophageal temperature monitoring during radiofrequency catheter ablation: Experimental study based on an agar phantom model}

Ignacio Rodríguez ${ }^{1}$, Juan L. Lequerica ${ }^{2}$, Enrique J. Berjano*,3, Maria Herrero², Fernando Hornero ${ }^{1}$

${ }^{1}$ Department of Cardiac Surgery, University General Hospital, Valencia, Spain

${ }^{2}$ Cardiac Research Laboratory, Instituto de Biomedicina, Spanish Council for Scientific Research (CSIC), Valencia, Spain

${ }^{3}$ Center for Research and Innovation on Bioengineering, Valencia Polytechnic University, Valencia, Spain

*Address for correspondence:

Dr. Enrique J. Berjano

Electronic Engineering Department, Valencia Polytechnic University.

Camino de Vera, 46022 Valencia, Spain.

E-mail: eberjano@eln.upv.es

Short title: Esophageal temperature monitoring during RF ablation 


\begin{abstract}
Although previous studies have established the feasibility of monitoring esophageal temperature during radiofrequency (RF) cardiac ablation using an esophageal temperature probe (ETP), some questions remain regarding its efficacy. The aims of this study were to study the effect of the location of the ETP on the temperature reached, and to test the characteristics of ETP as used in clinical practice. We constructed an agar phantom to model the thermal and electrical characteristics of the biological tissues (left atrium, esophagus, and connective tissue). The ETP was positioned at $6.5 \mathrm{~mm}$ from an ablation electrode and at distances of $0,5,10,15,20 \mathrm{~mm}$ from the catheter axis. A thermocouple was located on the probe to measure the actual temperature of the external esophageal layer during the ablations $\left(55^{\circ} \mathrm{C}, 60 \mathrm{~s}\right)$. The mean temperatures reached at the thermocouple were significantly higher than those measured by the ETP $\left(48.3 \pm 1.9^{\circ} \mathrm{C}\right.$ versus $\left.39.6 \pm 1.1^{\circ} \mathrm{C}\right)$. The temperature values measured with the ETP were significantly lower when the probe was located further from the catheter axis (up to $2.5^{\circ} \mathrm{C}$ lower when the distance from the probecatheter axis was $2 \mathrm{~cm}$. The dynamic calibration of the ETP showed a mean value for the time constant of $8 \mathrm{~s}$. In conclusion, the temperature measured by the ETP always underestimates the temperature reached in the thermocouple. This fact can be explained by the distance gap between thermocouple and probe and by the dynamic response of the ETP. The greater the distance between ETP and catheter axis the greater is the temperature difference.
\end{abstract}


Key words: Agar phantom, esophageal temperature probe, esophagus, left atrium, radiofrequency ablation, temperature measurement.

\section{Financial support}

This work was partially supported by the "Plan Nacional de Investigación Científica, Desarrollo e Innovación Tecnológica del Ministerio de Educación y Ciencia" of Spain (TEC 2005-04199/TCM) and by an R\&D contract (CSIC-20060633) between Edwards Lifescience Ltd. and the Spanish Council for Scientific Research (CSIC). 


\section{Introduction}

The use of radiofrequency (RF) in cardiology has advanced considerably in recent years. It has long been used in ablation of arrhythmias as a proven source of energy of demonstrated effectiveness and is broadly used in the field of electrophysiology. However, the use of this type of energy is not exempt from complications. Some years ago the first cases of atrioesophageal fistulae were reported during ablation procedures performed on the left atrium during cardiac surgery (Doll et al 2003, Gillinov et al 2001), and more recently during catheter ablation (Pappone et al 2004, Scanavacca et al 2004). Since then, there have been many attempts at avoiding this occasional but critical problem. It is known that the distance between the esophagus and the left atrium can be only a few millimeters (Hranitzky et al 2004, Lemola et al 2004) and this anatomic relationship is subject to wide variations. Some authors have tried to identify high risk patients by studying the atriumesophagus distance by means of image techniques (Hranitzky et al 2004, Lemola et al 2004, Sanchez-Quintana et al 2005). In some cases, fluoroscopy has been used during the ablation procedure to control the points where the distance is small (Cummings et al 2005, Perzanowski et al 2006). Other authors have preferred to work with lower power when ablating the more risky areas (Pappone et al 2004), although recent studies have suggested that power is not a predictor of the esophageal temperature, and in consequence, it is not useful for predicting esophageal lesions (Cummings et al 2005).

A strategy of increasing interest consists of monitoring the esophageal temperature during ablation by means of a temperature probe placed in the esophagus lumen (Cummings et al 2005, Perzanowski et al 2006, Redfearn et al 2005). In spite of its 
increasing acceptance, it has received a certain amount of criticism in recent reports (D'avila et al 2005, Meade et al 2005). It has been hypothesized that the type of probe, location in the correct plane, or close contact with the esophageal epithelium could be crucial for correct temperature monitoring and that these factors might not be considered in most cases. In fact, a previous computer modeling study showed that the incorrect location of the intraesophageal probe could significantly underestimate the maximum temperature presumably reached on the horizontal plane of the ablation catheter (Hornero and Berjano 2006). These first computational results encouraged us to develop an experimental model which simulates the circumstances that take place in this process with a high degree of accuracy. The aims of this study were 1) to analyze how the distance between ablation catheter and the esophageal temperature probe (ETP) affected the apparent esophageal temperature reached, and 2) to assess the limitations of the ETP employed in current clinical practice.

\section{Methods}

Previous computer modeling studies (Berjano and Hornero 2005, Hornero and Berjano 2006) have concluded that the distances between ablation electrode, esophagus, and temperature probe are critical factors in the study of temperature evolution during RF ablation. Moreover, it is very difficult to build an ex vivo model including fragments of esophagus, connective and cardiac tissue. For these reasons, we built a model based on an agar phantom. This methodology has been widely employed in experimental studies on RF ablation (Goldberg et al 2001, Lobo et al 2004, Solazzo et al 2005). It reduces variations in 
the electrical and thermal characteristics of ex vivo tissue to a minimum and provides control of the distances between the different elements of the model (such as ablation electrode and temperature probes).

\subsection{Construction of the phantom}

Figure 1 shows a diagram of the phantom and experimental setup. The agar phantom was constructed utilizing a methacrylate tube container with $50 \mathrm{~mm}$ external diameter and 30 mm in height. Before building the agar phantom, we placed a thermistor-based ETP perpendicular to the catheter axis (see Fig. 1A) and at $6.5 \mathrm{~mm}$ from the surface of the phantom. This distance value corresponds with the human anatomic distances previously reported (Ho et al 1999, Sanchez-Quintana et al 2005). The temperature probe employed was a 9 Fr esophageal/rectal probe 400 ER400-9 (Respiratory Support Products Inc., Tijuana Mexico) compatible with the YSI 400 series (YSI Temperature, Dayton, OH, USA). This type of probe is currently being employed to monitor the esophageal temperature during RF ablation (Perzanowski et al 2006, Redfearn et al 2005). The temperature probe was placed inside a simulated esophageal tube. A 22G $\mathrm{G}^{1 / 2}$ needle perpendicular either to the catheter axis or to the esophageal probe was also positioned in order to insert the appropriate temperature probe later. A solution of $5 \%$ agar in deionized water was previously heated over $100^{\circ} \mathrm{C}$. The methacrylate container was then filled with melted agar and allowed to solidify in a refrigerated environment at $4^{\circ} \mathrm{C}$ for at least 4 hours prior to use to ensure proper solidification and homogeneity. A T-type thermocouple microprobe IT-21 of $0.41 \mathrm{~mm}$ diameter (Physitemp Instruments, Crifton, NY, USA) was 
also situated on the surface of the ETP at the center of the phantom. For this, after cooling the phantom, the spinal needle was withdrawn and the microprobe was positioned manually through the canal formed in the gel.

\subsection{Experimental setup}

Figure $1 \mathrm{~B}$ shows a diagram of the experimental setup employed in this study. The phantom was submerged in a bath (polycarbonate container of $25 \times 20 \times 18 \mathrm{~cm}$ ) containing $12 \mathrm{~L}$ of 0.5\% $\mathrm{NaCl}$ solution as blood-equivalent solution (Cao et al 2000), and maintained at $37^{\circ} \mathrm{C}$ throughout the experiments with an immersion thermostat Microtherm 3000423 (JP Selecta, Abrera, Barcelona, Spain). Additionally, and in order to simulate blood flow inside the atrium, a roller pump Stockert-Shiley (Stöckert Shiley, Irvine, CA, USA) was used to irrigate both the ablation electrode and the surface of the agar model by re-circulating saline solution from the bath at a flow rate of $1 \mathrm{~L} / \mathrm{min}$. Saline was infused as a parallel laminar flow to the surface of the agar phantom through an $18 \mathrm{~mm}$ diameter tube, situated at $20 \mathrm{~mm}$ from the ablation catheter, which mimics blood flow inside the atrium (Cao et al 2000, Jain and Wolf 2000).

\subsection{Radiofrequency ablation}

The ablations were conducted using a Blazer II 7Fr/4 mm ablation catheter (Boston Scientific, Natiok, MA, USA). We used a methacrylate scaffold in order to accurately locate the axis of the catheter at the symmetry axis of the phantom, establishing a total distance of $6.5 \mathrm{~mm}$ between the point of RF application (i.e. the electrode tip) and the 
temperature measurement point (i.e. the thermocouple microprobe and the upper external surface of the esophageal temperature probe). The scaffold allowed the ablation electrode to apply a constant pressure of 20 grams on the agar, ensuring good reproducibility throughout the experiments. An ablation generator EPT-1000XP (EP Technologies, Boston, MA, USA) was utilized to deliver unmodulated $500 \mathrm{kHz}$ sinusoidal current between the ablation electrode and a large metallic plate $(20 \times 20 \mathrm{~cm})$ located on the bottom of the saline bath. The ablations were conducted by programming a constant temperature of $55^{\circ} \mathrm{C}$ at the electrode tip, simultaneously limiting the output power to $50 \mathrm{~W}$. The duration of the ablations was $60 \mathrm{~s}$ in all the experiments.

\subsection{Temperature measurement}

The tip of the thermocouple microprobe was always fixed at the catheter axis, on the surface of the esophageal probe, and at a constant distance of $6.5 \mathrm{~mm}$ from the point of application of the RF catheter. Also, in order to model different relative positions between the ETP and the catheter axis, the ETP was situated at different values of distance from the catheter axis: 20, 15, 10, 5 and 0 mm (see Fig. 2). At each position, the probe was pulled out (see the direction of open arrow in Fig. 2) and was situated at the desired distance (20, 15, 10, 5 and $0 \mathrm{~mm}$ distances from the catheter axis). We carried out five ablations for each position. The signals provided from the thermocouple and from the ETP were sent to a data acquisition system PowerLab 8sp (ADInstruments, Colorado Springs, CO, USA), sampled to $40 \mathrm{kHz}$, and digitalized with 16-bits resolution for subsequent analysis, which was performed using on-screen digital calipers using the software Chart v4.0.2 (ADInstruments, 
Colorado Springs, CC, USA). Finally, both systems designed to measure temperature were calibrated.

\subsection{Static and dynamic calibration of the esophageal temperature probe}

Like any other sensor, the performance characteristics of the ETP considered in this study can be broken down into the sub-areas of static and dynamic characteristics (Doebelin 1983). In our study, and prior to the ablation experiments, we first conducted a static calibration of the esophageal probe using a pre-heated water tank and a digital multimeter Fluke 77 (Fluke Corporation, Everett, WA, USA) with temperature-measuring option based on a K-type thermocouple ( $0.3 \%$ accuracy). We then conducted a two-points adjust via software in order to minimize offset and gain errors. As a consequence, the total accuracy of our temperature-measuring system based on the ETP was $\pm 0.5^{\circ} \mathrm{C}$.

Once the static characteristics were assessed, we conducted a dynamic calibration. Temperature sensors are classified as first-order systems, since their dynamic response is controlled by a first-order differential equation which describes the rate of heat transfer between the sensor and the surrounding medium (Dally et al 1984). In order to conduct the dynamic calibration, we considered the response of the esophageal probe to step-function inputs. This was done by suddenly immersing the probe in a medium maintained at a constant temperature of $\approx 75^{\circ} \mathrm{C}$. The time response followed an exponential function such as that shown in Fig. 3 (dotted line), which is a typical step response of a first-order system. In this kind of system the parameter "time constant" is defined, and is a measure of how quickly the system responds. It mathematically represents the time it takes the system's step 
response to reach about $63.2 \%$ of its final value. Additionally, we removed the plastic sheath from the esophageal probe (see Fig. 4) and conducted a dynamic calibration of the exposed thermistor (see dashed line in Fig. 3). The experiments were repeated ten times, and the "time constant" of each sensor type was expressed as mean \pm standard deviation.

\subsection{Statistical analysis}

The temperature values from thermocouple and ETP reached at $60 \mathrm{~s}$ of ablation were compared using a U-Mann-Whitney’s test. Differences in variables were considered to be significant at a threshold of $P<0.05$. Statistical analyses were performed with the software SPSS version 12.0 (SPSS, Chicago, IL, USA).

\section{Results}

\subsection{Effect of the location of esophageal probe on the temperature reached}

Figure 5 shows the temperature values reached at $60 \mathrm{~s}$ of ablation both in the thermocouple microprobe and in the esophageal probe. The temperature values measured with the thermocouple microprobe showed more dispersion and higher values $\left(48.3 \pm 1.9^{\circ} \mathrm{C}\right.$, ranging from 44.8 to $51.4^{\circ} \mathrm{C}$ ) than those measured by the esophageal probe $\left(39.6 \pm 1.1^{\circ} \mathrm{C}\right.$, ranging from 38.3 to $\left.41.5^{\circ} \mathrm{C}\right)(P<0.05)$. In fact, the differences between sensors ranged from 5.4 to $12.9^{\circ} \mathrm{C}$. On the other hand, the values measured by the esophageal probe were lower when the probe was located farther from the catheter axis $(P<0.05)$. The temperature reached was, in fact, more than $2.5^{\circ} \mathrm{C}$ lower when the distance between catheter axis and esophageal probe was $2 \mathrm{~cm}$ instead of $0 \mathrm{~cm}$. The delivered power ranged from 12 to $24 \mathrm{~W}$. 


\subsection{Lag time of the esophageal temperature probe}

The dynamic calibration of the ETP showed a time constant $\left(\tau_{\mathrm{P}}\right)$ of $8.0 \pm 0.6 \mathrm{~s}$. Technically, this means that when the temperature at the probe abruptly varied, it took a mean value of 8 seconds to change from the initial temperature of $\approx 36^{\circ} \mathrm{C}$ to $\approx 60.7^{\circ} \mathrm{C}$ (i.e. $63.2 \%$ of the difference between the initial temperature and the final temperature of $\approx 75^{\circ} \mathrm{C}$ ). From a clinical point of view, this fact indicates that the esophageal probe shows a significant delay in measuring. Likewise, we obtained a similar time evolution when we conducted the dynamic calibration of the uncovered esophageal probe (dashed line in Fig. 3). However, in this case the time constant was notably lower $\left(\tau_{\mathrm{TM}}=1.5 \pm 0.3 \mathrm{~s}\right)$. In other words, the plastic sheath of the probe undoubtedly interferes with the temperature register and implies a slower response.

\subsection{Effect of the dynamic response of the probe on temperature monitoring}

In order to assess the possible causes of the underestimation of temperature of the esophageal probe, we analyzed the five ablations conducted when the esophageal probe was located exactly at the catheter axis. During these ablations, the temperature in the thermocouple located on the esophageal probe remained unaltered during the first $7.5 \pm 2.0 \mathrm{~s}$ $(\mathrm{n}=5)\left(\mathrm{t}_{1}\right.$ in Fig. 6). It then increased and followed an approximately lineal evolution until reaching a temperature of $50.8 \pm 0.8^{\circ} \mathrm{C}(\mathrm{n}=5)$ at $60 \mathrm{~s}$ (Fig. 6). This lineal-like behavior leads us to suppose that the esophageal probe is being excited with a linear-function input, and as 
a consequence, since this probe is a first-order system with a $\tau_{\mathrm{P}} \approx 8 \mathrm{~s}$ (as previously calculated), it is possible to estimate the steady-state measurement error $\left(e_{m, s s}\right)$ due exclusively to its lag time. The dashed line in Fig. 6 shows the estimated time response (delayed $\tau_{\mathrm{P}}$ times) which involves a final temperature of $48.7^{\circ} \mathrm{C}$, i.e. an $\mathrm{e}_{\mathrm{m}, \mathrm{ss}}$ of $2.1^{\circ} \mathrm{C}$. However, this final temperature value is higher than those found in our set of experiments with the sensory zone of the probe located at the catheter axis $\left(41.1 \pm 0.3{ }^{\circ} \mathrm{C}, \mathrm{n}=5\right)$, which suggests that factors other than lag time could influence the total underestimation. These could include the distance between thermocouple and thermistor (previously commented on), and the fact that the esophageal probe is not being totally excited by a thermal linear function but only in its upper zone (i.e. on the side near to the ablation electrode).

\section{Discussion}

Ablation of the left atrium has now become an effective treatment for atrial fibrillation. It is still a developing technique, widely accepted, but not exempt from complications. The first reports of atrioesophageal fistulae come from the direct endocardial application of radiofrequency in a surgical field (Doll et al 2003, Gillinov et al 2001). Later, the first cases of fistulae secondary to percutaneous ablation were published (Pappone et al 2004, Scanavacca et al 2004). After years of clinical experience, it has been accepted that the incidence of this complication could be around $1 \%$ of ablation procedures in the left atrium (Cappato et al 2005). Some assumptions have been made about the main predictors of esophageal thermal lesion, such as type of catheter, presence of echocardiographic probe, area of the left auricle to be ablated and its anatomic relation with the esophagus 
(Piorkowski et al 2006, Tsao et al 2005). At the same time, clinical tests have been carried out to look for ways to avoid lesions. These have included image studies to anatomically detect high risk patients (Lemola et al 2004), modifications to the electrical parameters programmed in the RF generator (Doll et al 2003), and the esophageal temperature monitoring (Cummings et al 2005), a strategy that is becoming widely accepted.

Our study presents an experimental model to evaluate the effect of the position of the ETP during endocardial percutaneous RF ablation of the left atrium. Firstly, our results showed values of esophageal temperatures similar to those reported by previous clinical studies (Cummings et al 2005, Kuwahara et al 2005, Redfearn et al 2005) so that our model represents a close approximation to the clinical situation. Cummings et al (2005) reported, with similar ablation parameters (8-mm tetra-pole percutaneous catheter, and $55^{\circ} \mathrm{C}$ preset temperature), a range of esophageal temperature between 38.5 and $44^{\circ} \mathrm{C}$ in areas considered in proximity to the catheter ablation with a $4.2 \pm 2.1 \mathrm{~mm}$ distance between endocardium and esophageal lumen. We obtained a maximum temperature of $\approx 41^{\circ} \mathrm{C}$ with a $6.5 \mathrm{~mm}$ distance (when the probe was located at the catheter axis).

Secondly, in our experiments we observed a difference of $\approx 2.5^{\circ} \mathrm{C}$ between the temperatures measured with the esophageal probe at the catheter axis (most favorable location) and at $20 \mathrm{~mm}$ distance from the catheter axis. This result clearly implies that a lateral displacement of the esophageal probe can lead to underestimating the temperature, which had previously been suggested by Perzanowsky et al (2006). This result also confirms the validity of the previous results obtained from a computer modeling study (Hornero and Berjano 2006). However, these computer results (using a distance between 
electrode and esophageal lumen of $5 \mathrm{~mm}$ ) showed a higher difference (up to $7^{\circ} \mathrm{C}$ ) (Hornero and Berjano 2006). The reasons for this disagreement might be the difference in electrodeesophagus distance (larger in this study), but we believe that it is principally due to the characteristics of the esophageal probe itself, which has considerable volume (i.e. high thermal inertia), which could significantly affect the measurement.

We also observed that the maximum temperatures reached at the esophageal probe and at the thermocouple when they were aligned at the same point with respect to the catheter axis differed by a mean value of $9.7^{\circ} \mathrm{C}$, despite being in close contact. This temperature gap could be explained by the structure of the esophageal probe. As can be observed in Fig. 4, it has a plastic cover that contains the temperature sensor (i.e. the thermistor). Both the plastic cover (with a significant thickness), and the air surrounding the thermistor, cause considerable separation of thermocouple and thermistor, so that they are located at different distances from the catheter, and hence record different temperature values. From a clinical point of view, this finding suggests that the temperature measured inside the esophagus lumen could be much lower than the real temperature reached in the external esophagus wall. In conclusion, the structure of the esophageal probe itself implies a significant static error. Moreover, since we employed an small probe (9 Fr pediatric), this static error might even be higher with adult probes (10-12 Fr) due to higher gap between the esophagus lumen and thermistor.

However, the same structure also involves a dynamic error. To be more precise, the elevated value of time constant obtained from the dynamic calibration of the esophageal probe suggests that the underestimation in measuring the temperature using this probe 
might be partially due to its time constant. In addition, it is known that the value of the steady-state measurement error $\left(\mathrm{e}_{\mathrm{m}, \mathrm{ss}}\right)$ is not only directly proportional to $\tau_{\mathrm{P}}$, but also increases directly with the rate of change of the measured temperature (Doebelin 1983). Since it is known that the temperature at points close to the ablation electrode increases faster than those at a greater distance (Cao et al 2000, Wittkampf et al 1996), the measuring error might be higher in these cases. Thus, the problem associated with the lag time of the esophageal probe is more hazardous when the probe is closer to the ablation electrode. This problem has also been previously reported by other authors (Teplitsky et al 2005). The value of the time constant could be improved (i.e. reduced) by designing a temperature sensor with a small mass, e.g. the very small thermocouple employed in this study has a constant time of $\approx 40$ ms (see solid line in Fig. 3). In conclusion, new technologies for safe and accurate monitoring of the esophageal temperature should be designed to minimize the time response, and would probably include several small temperature sensors.

\subsection{Limitations of the study}

In our experimental set-up, we only considered the shift of the esophageal probe along an axis. On the other hand, it is known that in spite of positioning the probe at the level of the ablation catheter the esophagus lumen permits lateral displacements of the esophageal probe in any direction on a transversal plane (Perzanowski et al 2006). However, our previous modeling study showed similar temperature values when the measuring points were shifted the same distance from the catheter axis but in different directions (Hornero 
and Berjano 2006), which suggests that the experimental results obtained might also be valid under other shift conditions.

Finally, our set-up was based on a uniform agar model. To date, this technique has been widely employed in experimental studies on RF ablation for the treatment of tumor in homogeneous tissue (e.g. liver and kidney). We believe that our model correctly mimics heat transfer through multiple distinct tissue types and tissue interface layers (e.g. atrial myocardium, pericardium connective tissue, esophageal muscle) since that all these tissues offer similar values both of thermal and electrical characteristics.

\section{Conclusions}

Our experimental findings suggest that:

1) The temperature measured with the esophageal probe always underestimates the temperature reached in the thermocouple, which could be equivalent to the temperature of the esophagus. This fact can be partially explained by the short distance between thermocouple and esophageal probe and by the slow dynamic response of the clinical probe.

2) Likewise, the increase in the distance between the sensory zone of the esophageal probe and the catheter axis underestimate the temperature reached in the thermocouple.

3) The rapid increase of the temperature reached in the thermocouple suggests that the signal obtained from an ETP should be sampled using a high frequency as opposed to those currently employed (1 sample each 5 s). 


\section{Acknowledgments}

We would like to thank the $\mathrm{R}+\mathrm{D}+\mathrm{i}$ Linguistic Assistance Office at the Universidad Politécnica of Valencia for their help in revising this paper. 

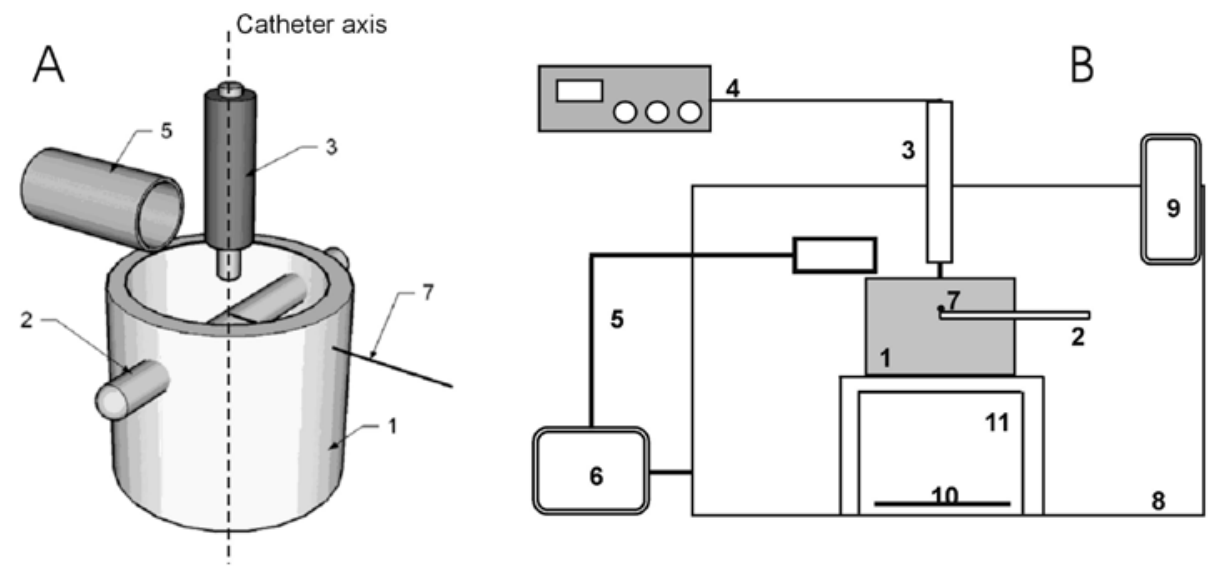

Figure 1 A: Diagram of the agar phantom model. B: Experimental setup of the agar phantom model: (1) agar phantom container, (2) esophageal temperature probe, (3) ablation catheter, (4) radiofrequency generator, (5) hydraulic circuit to simulate blood flow, (6) pump, (7) thermocouple microprobe, (8) saline bath at $37^{\circ} \mathrm{C}$, (9) immersion thermostat, (10) metallic plate, and (11) plastic frame. 


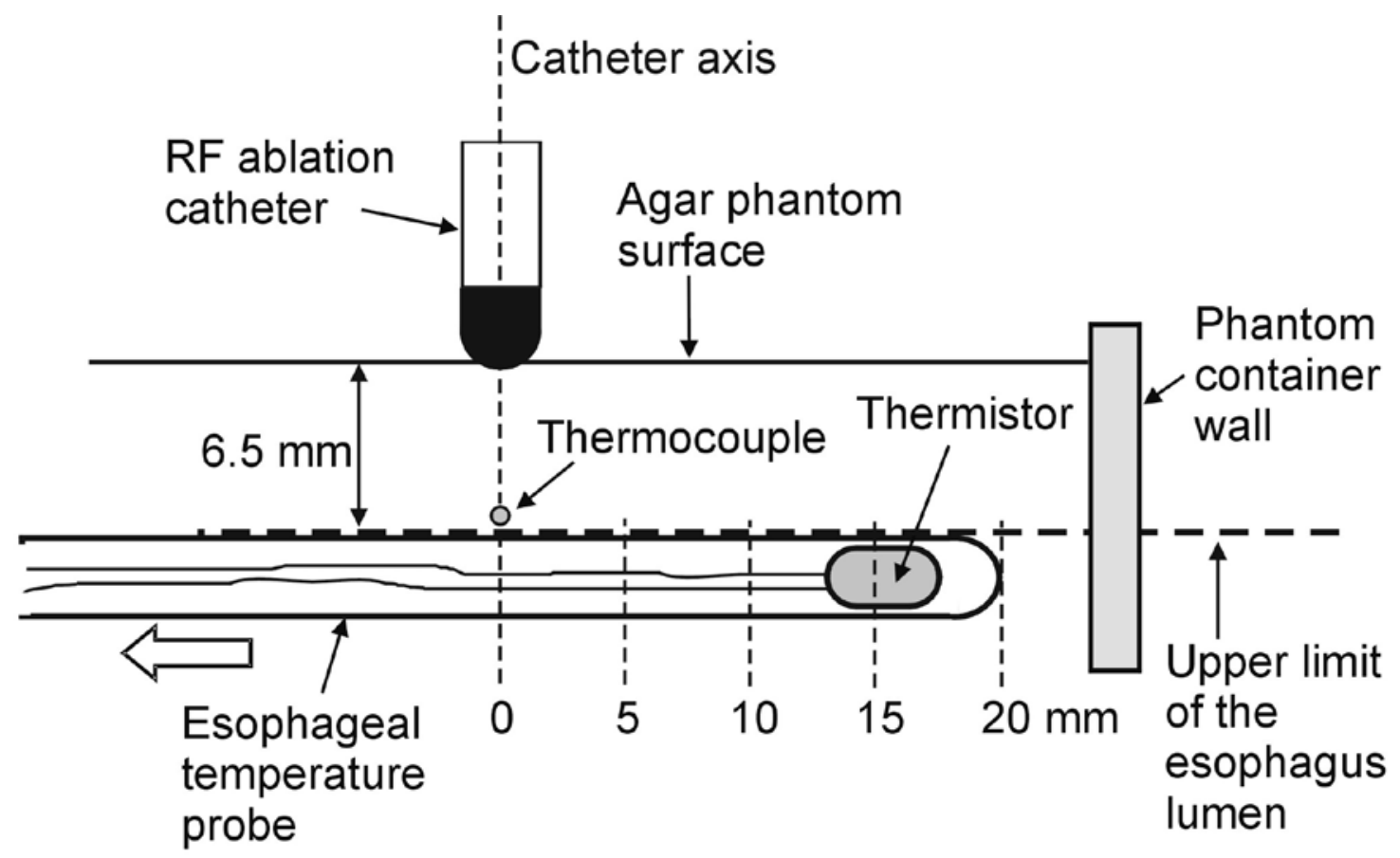

Figure 2 Relative position of the radiofrequency ablation catheter, the thermistorbased esophageal temperature probe and the thermocouple in the agar phantom model. The esophageal probe was slid in the direction of the open arrow to situate the middle point of the thermistor at the appropriate distance for the experiment (i.e. 20, 15, 10, 5 and $0 \mathrm{~mm}$ ) with reference to the catheter axis. 


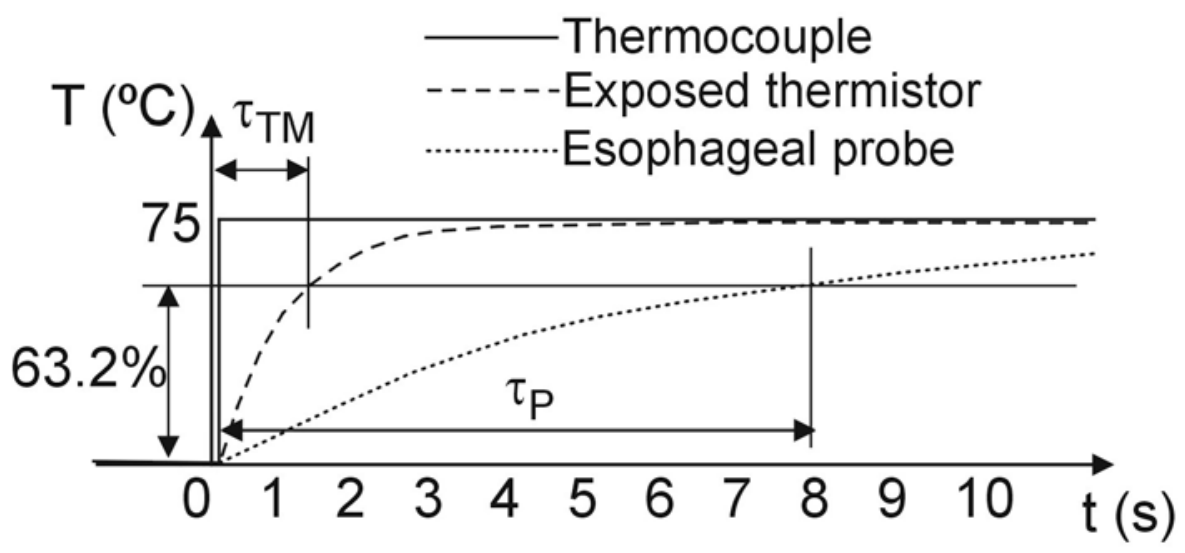

Figure 3 Step responses of esophageal temperature probe, uncovered esophageal temperature probe (i.e. with exposed thermistor) and thermocouple. The time constant of esophageal temperature probe $\left(\tau_{\mathrm{P}}\right)$ and the exposed thermistor ( $\left.\tau_{\mathrm{TM}}\right)$ was respectively of $8.0 \pm 0.6 \mathrm{~s}$ and $1.5 \pm 0.3 \mathrm{~s}$. The thermocouple showed a time constant of $\approx 40 \mathrm{~ms}$, and thus was employed as control signal to check the step waveform of the temperature. 


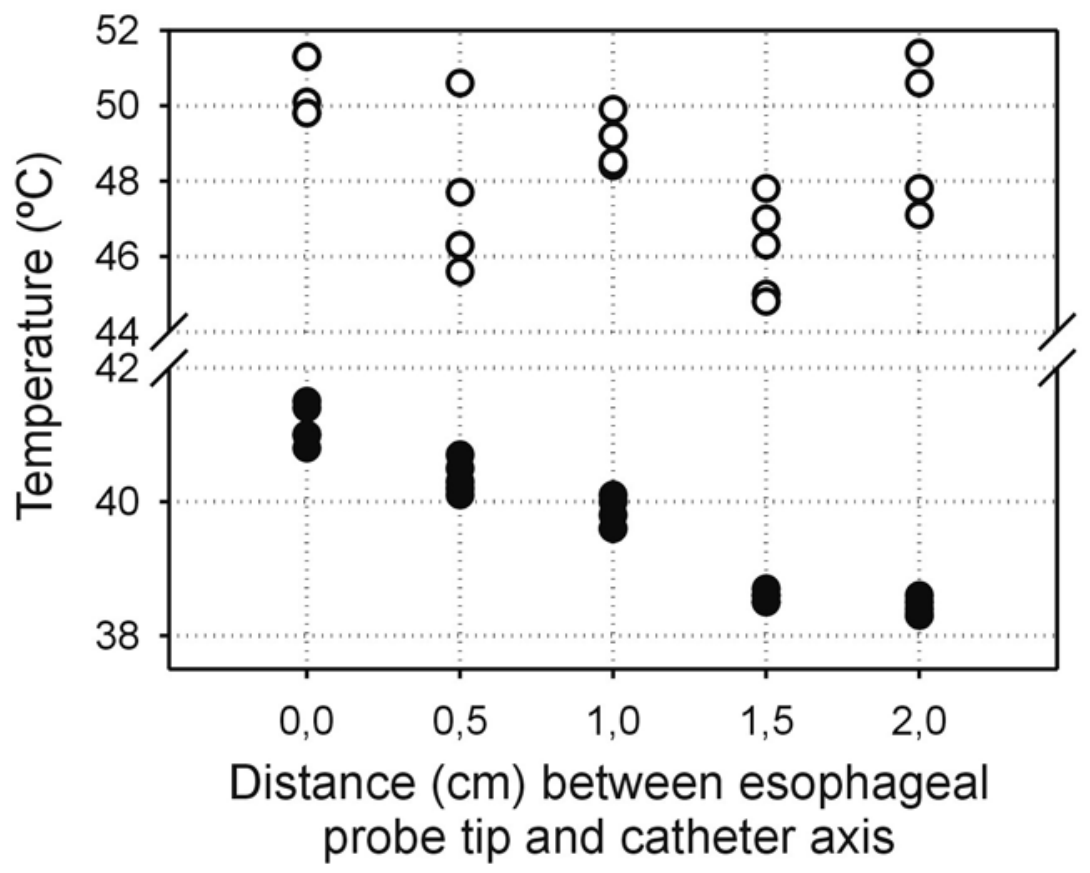

Figure 4 Photo showing the inner part of the thermistor-based esophageal temperature probe (9 Fr, YSI 400 series). A section of the plastic sheath has been removed in order to show the exposed thermistor (arrow). 


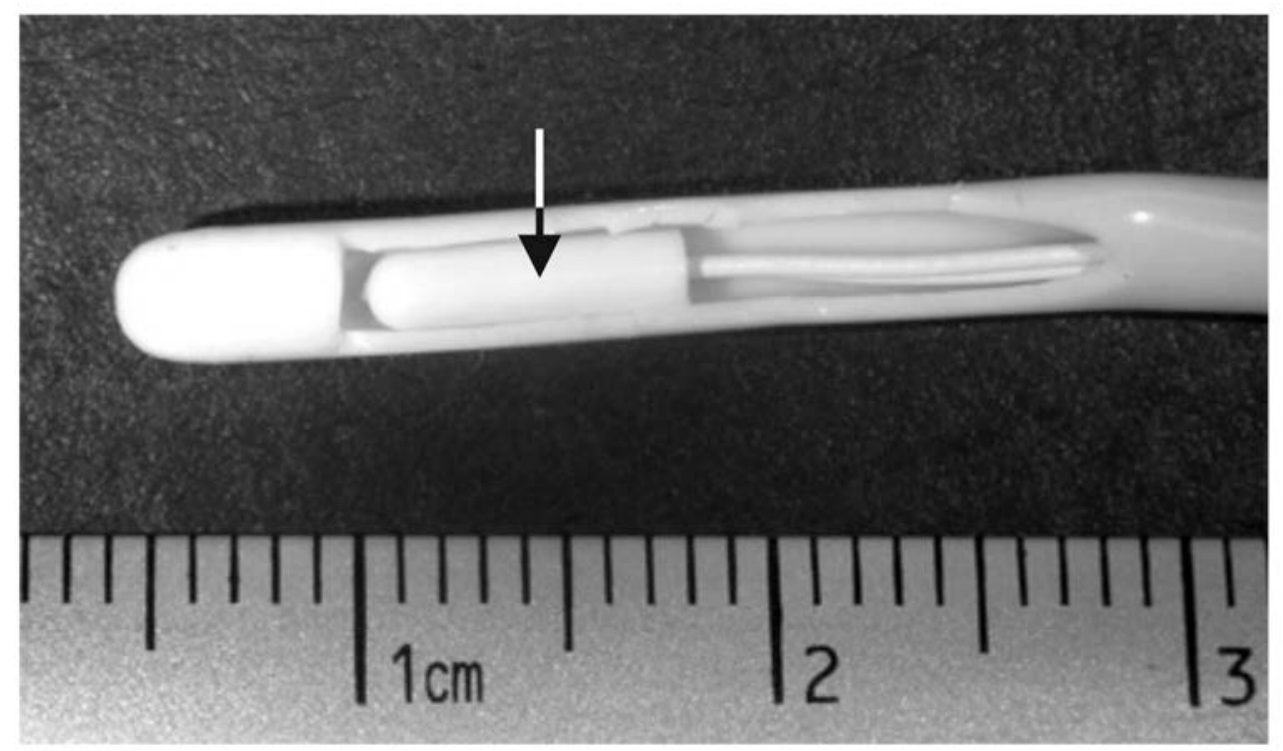

Figure 5 Values of temperature reached at $60 \mathrm{~s}$ of ablation in the thermocouple (open circle) and in the esophageal temperature probe (closed circle) for different locations in relation to the catheter axis. The thermocouple microprobe was always located on the catheter axis. 


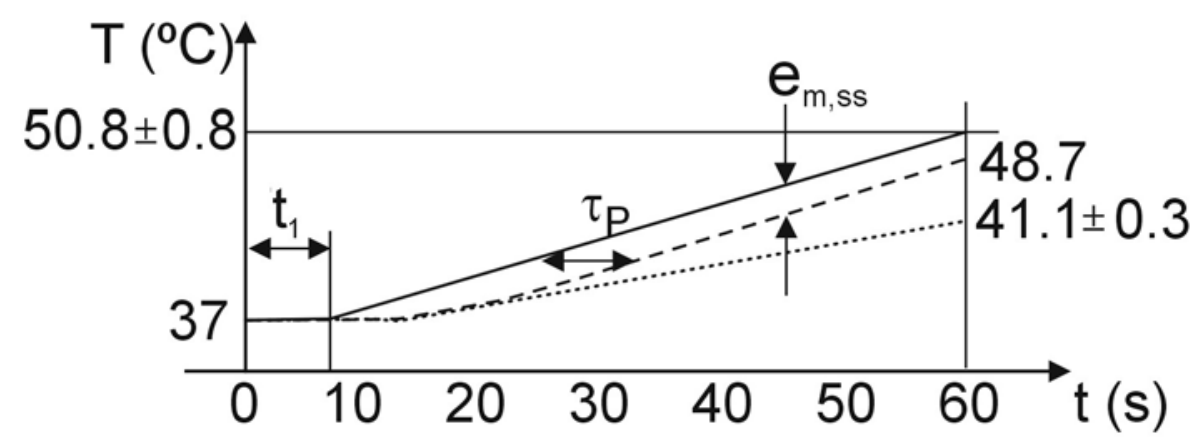

Figure 6 Time evolution of the temperature measured in the thermocouple (solid line) and in the esophageal temperature probe located at the catheter axis (dotted line). The final temperatures reached were $50.8 \pm 0.8^{\circ} \mathrm{C}$ and $41.1 \pm 0.3^{\circ} \mathrm{C}$ respectively. The time evolution of thermocouple was approximately a linear response beginning at $t_{1}=7.5 \pm 2.0 \mathrm{~s}$. Taking only the time constant of the esophageal probe $\left(\tau_{\mathrm{P}}\right)$ into account, the temperature reached in the probe would be $48.7^{\circ} \mathrm{C}$ (dashed line). 


\section{References}

Berjano E J and Hornero F 2005 What affects esophageal injury during radiofrequency ablation of the left atrium? An engineering study based on finite-element analysis Physiol. Meas. 26 837-848

Cao H, Vorperian V R, Tsai J Z, Tungjitkusolmun S, Woo E J and Webster J G 2000, Temperature measurement within myocardium during in vitro RF catheter ablation IEEE Trans. Biomed. Eng. 47 $1518-1524$

Cappato R, Calkins H, Chen S A, Davies W, Iesaka Y, Kalman J, Kim Y H, Klein G, Packer D and Skanes A 2005 Worldwide survey on the methods, efficacy, and safety of catheter ablation for human atrial fibrillation Circulation 111 1100-1105

Cummings J E, Schweikert R A, Saliba W I, Burkhardt J D, Brachmann J, Gunther J, Schibgilla V, Verma A, Dery M, Drago J L, Kilicaslan F and Natale A 2005 Assessment of temperature, proximity, and course of the esophagus during radiofrequency ablation within the left atrium Circulation 112 459-464

D'avila A, Maldonado P, Veronese F, Mendoça M L, Colafranceschi A S, Colle S and Saad E B 2005 Accuracy of esophageal temperature measurement and its correlation to microbubbles formation during catheter ablation of atrial fibrillation Heart Rhythm 2 S9

Dally J W, Riley W F and McConnell K G 1984 Instrumentation for engineering measurements (John Wiley \& Sons, New York)

Doebelin E O 1983 Measuring systems. Application and design (McGraw-Hill, New York)

Doll N, Borger M A, Fabricius A, Stephan S, Gummert J, Mohr F W, Hauss J, Kottkamp H and Hindricks G 2003 Esophageal perforation during left atrial radiofrequency ablation: Is the risk too high? J. Thorac. Cardiovasc. Surg. 125 836-842

Gillinov A M, Pettersson G and Rice T W 2001 Esophageal injury during radiofrequency ablation for atrial fibrillation J. Thorac. Cardiovasc. Surg. 122 1239-1240

Goldberg S N, Ahmed M, Gazelle G S, Kruskal J B, Huertas J C, Halpern E F, Oliver B S and Lenkinski R E 2001 Radio-frequency thermal ablation with $\mathrm{NaCl}$ solution injection: effect of electrical conductivity on tissue heating and coagulation-phantom and porcine liver study Radiology 219 157-165

Ho S Y, Sanchez-Quintana D, Cabrera J A and Anderson R H 1999 Anatomy of the left atrium: implications for radiofrequency ablation of atrial fibrillation J. Cardiovasc. Electrophysiol. 10 1525-1533

Hornero F and Berjano E J 2006 Esophageal temperature during radiofrequency-catheter ablation of left atrium: a three-dimensional computer modeling study J. Cardiovasc. Electrophysiol. 17 405-410 
Hranitzky P M, Contrafatto I, Kim R, Ashar M S and Bahnson T D 2004 MRI identifies significant variability in the anatomic relationship between the esophagus and left atrium in patients undergoing catheter ablation for atrial fibrillation Circulation 110 III-418

Jain M K and Wolf P D 2000 In vitro temperature map of cardiac ablation demonstrates the effect of flow on lesion development Ann. Biomed. Eng. 28 1066-1074

Kuwahara T, Takahashi A, Yokoyama Y, Kobori A, Sato A, Iesaka Y, Hirao K, Isobe M and Aonuma K 2005 Importance of esophageal temperature monitoring for the avoidance of esophageal injury during circumferential left atrial ablation Heart Rhythm 2 S156

Lemola K, Sneider M, Desjardins B, Case I, Han J, Good E, Tamirisa K, Tsemo A, Chugh A, Bogun F, Pelosi F Jr, Kazerooni E, Morady F and Oral H 2004 Computed tomographic analysis of the anatomy of the left atrium and the esophagus: implications for left atrial catheter ablation Circulation 110 3655-3660

Lobo S M, Afzal K S, Ahmed M, Kruskal J B, Lenkinski R E and Goldberg S N 2004 Radiofrequency ablation: modeling the enhanced temperature response to adjuvant $\mathrm{NaCl}$ pretreatment Radiology 230 175-182

Meade T, Razavi M, Yang D, Delapasse S, Donsky A, Ai T, Steiner P and Cheng J 2005 Real-time esophageal thermal profile during posterior left atrial radiofrequency ablation Heart Rhythm 2 S236

Pappone C, Oral H, Santinelli V, Vicedomini G, Lang C C, Manguso F, Torracca L, Benussi S, Alfieri O, Hong R, Lau W, Hirata K, Shikuma N, Hall B and Morady F 2004 Atrio-esophageal fistula as a complication of percutaneous transcatheter ablation of atrial fibrillation Circulation 109 2724-2726

Perzanowski C, Teplitsky L, Hranitzky P M and Bahnson T D 2006 Real-time monitoring of luminal esophageal temperature during left atrial radiofrequency catheter ablation for atrial fibrillation: observations about esophageal heating during ablation at the pulmonary vein ostia and posterior left atrium J. Cardiovasc. Electrophysiol. 17 166-170

Piorkowski C, Hindricks G, Schreiber D, Tanner H, Weise W, Koch A, Gerds-Li J H and Kottkamp $\mathrm{H} 2006$ Electroanatomic reconstruction of the left atrium, pulmonary veins, and esophagus compared with the "true anatomy" on multislice computed tomography in patients undergoing catheter ablation of atrial fibrillation Heart Rhythm 3 317-327

Redfearn D P, Trim G M, Skanes A C, Petrellis B, Krahn A D, Yee R and Klein G J 2005 Esophageal temperature monitoring during radiofrequency ablation of atrial fibrillation $J$. Cardiovasc. Electrophysiol. 16 589-593

Sanchez-Quintana D, Cabrera J A, Climent V, Farre J, Mendonca M C and Ho S Y 2005 Anatomic relations between the esophagus and left atrium and relevance for ablation of atrial fibrillation Circulation 112 1400-1405 
Scanavacca M I, D'avila A, Parga J and Sosa E 2004 Left atrial-esophageal fistula following radiofrequency catheter ablation of atrial fibrillation J. Cardiovasc. Electrophysiol. 15 960-962

Solazzo S A, Liu Z, Lobo S M, Ahmed M, Hines-Peralta A U, Lenkinski R E and Goldberg S N 2005 Radiofrequency ablation: importance of background tissue electrical conductivity-an agar phantom and computer modeling study Radiology 236 495-502

Teplitsky L, Perzanowski C, Durrani S, Berman A E, Hranitzky P M and Bahnson T D 2005 Radiofrequency catheter ablation for atrial fibrillation produces delayed and long lasting elevation of luminal esophageal temperature independent of lesion duration and power Heart Rhythm 2 S8-S9

Tsao H M, Wu M H, Higa S, Lee K T, Tai C T, Hsu N W, Chang C Y and Chen S A 2005 Anatomic relationship of the esophagus and left atrium: implication for catheter ablation of atrial fibrillation Chest 128 2581-2587

Wittkampf F H, Nakagawa H, Yamanashi W S, Imai S and Jackman W M 1996 Thermal latency in radiofrequency ablation Circulation 93 1083-1086 